\title{
Numerical Modelling of Flexible Heaving Foils
}

\author{
Dmitry Kolomenskiy, ${ }^{\mathrm{a}, *}$ Thomas Engels ${ }^{\mathrm{b}, \mathrm{c}}$ and Kai Schneider ${ }^{\mathrm{b}}$ \\ ${ }^{a}$ McGill University / CRM, Montréal, QC, Canada \\ ${ }^{\mathrm{b}}$ M2P2-CNRS and Aix-Marseille Université, France \\ ${ }^{c}$ ISTA, TU Berlin, Germany
}

\begin{abstract}
We consider the effects of chordwise flexibility on the aerodynamic performance of flapping wings using numerical simulation. The twodimensional Navier-Stokes equations are solved using a Fourier pseudo-spectral method with no-slip boundary conditions imposed by the volume penalization method. The flexible wing is modelled with a non-linear beam equation. Our numerical simulations of heaving plates show that the maximum thrust is achieved at a stroke frequency lower than resonant, which is in agreement with experiments. The oscillatory part of the force only increases in amplitude when the frequency increases. We also consider aerodynamic interactions between two heaving foils.
\end{abstract}

Index Terms-Flapping flight, fluid-structure interaction, spectral method, volume penalization method.

\section{INTRODUCTION}

In forward flight, flapping wings generate the desired thrust by doing up and down strokes combined with supination and pronation movements that ensure the required angle of attack. A canonical two-dimensional approximation to this process is the heaving (plunging) and pitching motion of a foil, shown in Fig. 1. If the three-dimensional wing structure is rigid, the time evolution of the positional angle $\varphi(t)$ and the angle of incidence $\alpha(t)$ should be imposed (Fig. 1a). In the twodimensional approximation, shown in Fig. 1(b), this is equivalent to prescribing a heave motion $y_{l e}(t)$ and a pitch motion $\alpha(t)$ (see, e.g., [1]). However, if the wing is flexible and clamped at the leading edge, the effective angle of incidence varies passively due to the action of inertial and aerodynamic forces (Fig. 1c), and this mechanism also generates thrust with only the heave motion imposed (see, e.g, [6,7]). Note that there are several distinct effects associated with the spanwise and the chordwise flexibility [7,9], and this work

\footnotetext{
* Corresponding author: Burnside Hall, Room 1131 805 Sherbrooke W., Montreal, QC, H3A 2K6, Canada E-mail: dkolom@gmail.com
}

focuses on the chordwise flexibility only.

In this paper, we present a new computational method for modelling the flow dynamics around flexible foils, and show its application to flapping wing propulsion. The computational setup and the governing equations are described in Section II, followed by an outline of the discretization schemes of the fluidstructure solver in Section III. The results of the numerical simulations of single and double flapping wings are discussed in Section IV. Conclusions are drawn in Section V.
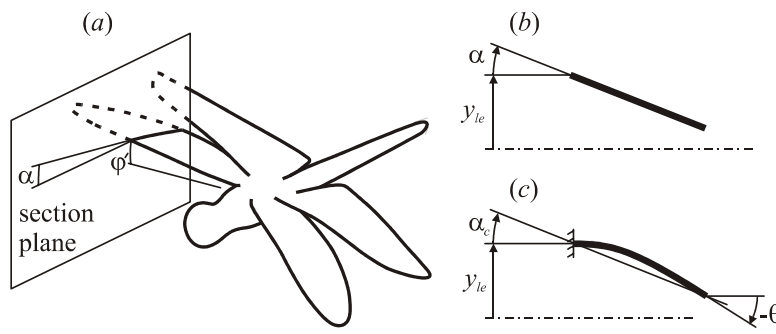

(c)

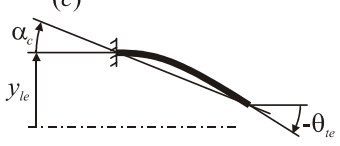

Fig. 1. Schematic diagram showing a chordwise section of a three-dimensional wing $(a)$, a two-dimensional approximation to a rigid pitching and heaving foil $(b)$ and a flexible heaving foil clamped at the leading edge $(c)$. The following geometric parameters are shown: $\varphi$ - positional angle, $\alpha$ - geometric angle of attack, $y_{l e}$ - vertical coordinate of the leading edge, $\theta_{t e}$ - deflection angle at the trailing edge, $\alpha_{c}$ - geometric angle of attack of a flexible foil.

\section{COMPUTATIONAL SETUP AND GOVERNING EQUATIONS}

The computational approach extends our previous work on the modelling of flows past rigid obstacles $[2,3]$. We consider either one or two flexible flat wings immersed in viscous incompressible fluid, see Fig. 2. The flow is assumed to be two-dimensional. The motion of the fluid is described by the Navier-Stokes equations. We employ the vorticity-stream function formulation. For convenience, all equations and quantities are rendered dimensionless, with the fluid density, the wing chord length and a given velocity scale being the set of reference values. The NavierStokes equations are solved with the no-slip boundary condition $\left.\mathbf{u}\right|_{\Gamma}=\left.\mathbf{u}_{s}\right|_{\Gamma}$ imposed at the wing surface $\Gamma$. 
We use the volume penalization method [5] to model the no-slip boundary condition, yielding

$$
\frac{\partial \omega}{\partial t}+\mathbf{u} \cdot \nabla \omega-\frac{1}{R e} \Delta \omega=-\nabla \times\left(\frac{\chi}{\varepsilon}\left(\mathbf{u}-\mathbf{u}_{s}\right)\right)-\frac{\chi_{\text {sponge }}}{\varepsilon} \omega,
$$

where $\omega=\nabla \times \mathbf{u}$ is the vorticity, the velocity $\mathbf{u}$ is determined as $\mathbf{u}=\nabla^{\perp} \psi$ (the mean flow is zero in the present work), $\nabla^{\perp}=(-\partial / \partial y, \partial / \partial x)^{T}$ denotes the orthogonal gradient, and the stream function $\psi$ is the solution of $\nabla^{2} \psi=\omega$. The parameter $R e$ is the Reynolds number based on the wing chord, the chosen reference velocity and the kinematic viscosity of the fluid. The first term on the right-hand side serves to impose the no-slip boundary condition. It contains the mask function $\chi(\mathbf{x}, t)$, which is the indicator function equal to 1 inside the domain occupied by the solid wing and 0 elsewhere. The field $\mathbf{u}_{\mathrm{s}}(\mathbf{x}, t)$ is the velocity of the solid plate. The parameter $\varepsilon$ is a small number, physically interpreted as the permeability of the solid. The solution of the penalized problem converges to the solution of the original Dirichlet boundary-value problem as $\varepsilon \rightarrow 0$ [5]. The second term on the right-hand side is a 'vorticity sponge' introduced in order to remove the vorticity from the outer boundary of the computational domain. As explained in the next section, our numerical method assumes periodic boundary conditions, therefore we have to ensure that the vortex wake does not re-enter the computational domain after crossing its boundary. Thus, the sponge mask function $\chi_{\text {sponge }}$ is equal to 1 in a thin layer near the outer boundary and 0 elsewhere.

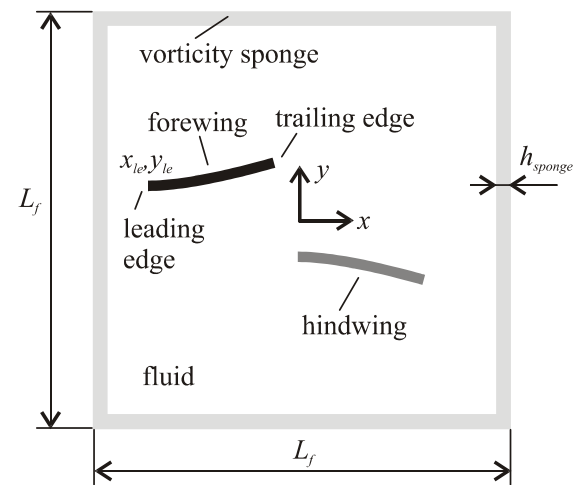

Fig. 2. Computational setup. In the present numerical simulations, $L_{f}=8$ and $h_{\text {sponge }}=0.05$.

The wing section is modelled as an inextensible beam made of a linear-elastic material satisfying Hooke's law. The structural contribution to the energy dissipation is neglected. The stiffness and mass are distributed uniformly. Assuming at this point that the beam is slender, we employ a non-linear beam equation suitable for large deflections,

$$
\begin{aligned}
& \frac{\partial^{2} T}{\partial s^{2}}-T\left(\frac{\partial \theta}{\partial s}\right)^{2}=-[p] \frac{\partial \theta}{\partial s}-2 \eta \frac{\partial \theta}{\partial s} \frac{\partial^{3} \theta}{\partial s^{3}}-\eta\left(\frac{\partial^{2} \theta}{\partial s^{2}}\right)^{2}-\mu\left(\frac{\partial \theta}{\partial t}\right)^{2} \\
& \mu \frac{\partial^{2} \theta}{\partial t^{2}}=-\frac{\partial[p]}{\partial s}-\eta \frac{\partial^{4} \theta}{\partial s^{4}}+\left(T+\eta\left(\frac{\partial \theta}{\partial s}\right)^{2}\right)^{2} \frac{\partial^{2} \theta}{\partial s^{2}}+2 \frac{\partial T}{\partial s} \frac{\partial \theta}{\partial s}
\end{aligned}
$$

where $T$ is the tension inside the beam and $\theta$ is the local deflection angle. The wing has unit chord length, and $s \in[0,1]$ is the reduced arc length coordinate measured from the leading edge. The parameters $\mu$ and $\eta$ are, respectively, the reduced density and stiffness of the beam. The pressure difference between the upper and the lower side, $[p]$, is obtained by interpolating the fluid pressure on the beam surface defined as an isoline of the mask function $\chi=0.5$. The clamped-free boundary conditions for (2)-(3) are formulated as

$$
\begin{gathered}
\theta=0, \frac{\partial T}{\partial s}+\eta \frac{\partial^{2} \theta}{\partial s^{2}} \frac{\partial \theta}{\partial s}=0, T \frac{\partial \theta}{\partial s}-\eta \frac{\partial^{3} \theta}{\partial s^{3}}=[p]+\frac{\mathrm{d}^{2} y_{l e}}{\mathrm{~d} t^{2}} \text { at } s=0 \\
T=0, \frac{\partial \theta}{\partial s}=0, \frac{\partial^{2} \theta}{\partial s^{2}}=0 \text { at } s=1 .
\end{gathered}
$$

\section{NUMERICAL METHOD}

Equation (1) is solved using a classical Fourier pseudo-spectral method. In addition to its ease of implementation, this method provides an efficient way of solving the Poisson equation for the stream function $\psi$, since the Laplace operator is diagonal in the Fourier basis. Moreover, it employs the Fast Fourier Transform (FFT), for which there exist efficient and well optimized software packages. Meanwhile, the Fourier pseudo-spectral discretization implies that the computational domain is rectangular, the discretization grid is uniform Cartesian, and that the boundary conditions are periodic. Since the present study requires boundary conditions other than periodic, we use the volume penalization techniques to impose the no-slip conditions at the solid boundary and the vorticity sponge in the far field, as explained in the Section II. The time integration is exact for the viscous term and an adaptive second order Adams-Bashforth scheme is used for the nonlinear term. The details of the fluid solver were reported earlier in [2,3].

Equations (2)-(3) are solved using a second order finite difference spatial discretization. All derivatives in $s$ are approximated by central finite differences everywhere, except near the boundaries where we use fourth order backward schemes. To advance in time we apply a second order Crank-Nicolson scheme, and treat all terms semi-implicitly. To solve the resulting nonlinear algebraic system, Newton's method is applied with the Jacobian computed analytically.

The fluid-structure coupling is achieved by using a sequential staggered scheme. First, the fluid is advanced, while keeping the mask function $\chi$ unchanged during the time step. The updated fluiddynamic pressure is used to calculate the forces on the beam at the new time step, which are then transmitted to the solid solver to advance the beam to the next time level and to generate the mask function for the next time step. More details on the fluid-structure coupling method can be found in [4]. 


\section{RESULTS AND DISCUSSION}

\section{A. Single wing section}

The numerical simulations presented in this paper are inspired by experiments with a mechanical flapper model conducted by Ramananarivo et al. [6]. The model has two semi-circular wings with length $R=0.06 \mathrm{~m}$ and mid-span chord length $c=0.03 \mathrm{~m}$. The wing model chosen here as a reference has rigidity $B=1.83 \cdot 10^{-4} \mathrm{~N} \cdot \mathrm{m}$, mass per unit area $m_{s}=10.63 \cdot 10^{-2}$ $\mathrm{kg} \cdot \mathrm{m}^{-2}$, thickness $h=0.078 \mathrm{~mm}$ and relaxation frequency $f_{0}=34.2 \mathrm{~Hz}$. Since the far-field inflow velocity is zero, it cannot be used as a reference velocity necessary for normalizing the equations. Therefore, the reference velocity is set to $U_{r e f}=1 \mathrm{~m} / \mathrm{s}$, which is related to the wing kinematics as explained later in this Section. This corresponds to the following values of the dimensionless parameters of our computational setup:

$$
\eta=\frac{B}{c^{3} \rho_{f} U_{r e f}^{2}}=5.5284 \text { and } \mu=\frac{m_{s}}{c \rho_{f}}=2.8902,
$$

where it is assumed that the air density is $\rho_{f}=1.226$ $\mathrm{kg} / \mathrm{m}^{3}$.

The experimental value of the Reynolds number, $R e=c U_{\text {ref }} / v_{f}$, is about $2000\left(v_{f}\right.$ is the kinematic viscosity of air). Note that this value is based on the reference velocity $U_{r e f}$, as it enters equation (1). To obtain the Reynolds number based on the wing velocity, this value should be corrected by a factor proportional to the stroke frequency. Hence, matching the experimental Re presents difficulties for the numerical simulation if the frequency is large. For this reason, numerical simulations reported in this paper correspond to $R e=100$, that allows analyzing a wide range of frequencies. Meanwhile, similarities between the present simulations and earlier experimental findings [6] suggest that the Reynolds number effects are not dominant in this particular problem.

The thickness of the wing imposed in the fluid solver using the volume penalization method is determined from numerical considerations [4], and the penalization parameter equals $\varepsilon=2 \cdot 10^{-4}$. The periodic domain is a square of side $L_{f}=8$, and the vorticity sponge of strength $\varepsilon_{\text {sponge }}=2 \cdot 10^{-4}$ is applied at the four sides in thin layers with thickness $h_{\text {sponge }}=0.05$. We remind that, unless otherwise stated, all distances reported in this paper are normalized by the chord length $c$, time is normalized by $c / U_{\text {ref. }}$. The forces are per unit span and normalized by $\rho_{f} U_{r e f}^{2} c$. The number of discretization grid points in the fluid solver is equal to $N_{f}^{2}=2940^{2}$. In the beam equation solver, the number of points is $N_{s}=384$.

Initially, at time $t=0$, the fluid is at rest. Zero mean fluid velocity is imposed at all time $t$. During the simulation, the position of the leading edge of the foil varies in time as $x_{l e}=x_{0}$ and $y_{l e}=y_{0}+y_{F} \cos \left(2 \pi t / t_{F}\right)$, where the amplitude of the leading edge motion is $y_{F}=0.5$, the neutral position of the leading edge is $x_{0}=-1.5, y_{0}=0$, measured from the centre of the domain, and $t_{F}$ is the period of the sinusoidal motion. Note that the reference velocity $U_{r e f}$ corresponds to the maximum dimensional velocity of the leading edge oscillating with $t_{F}=\pi$. A series of simulations has been carried out with $t_{F}$ ranging from 1 to 6 . For $t_{F}=1.4$, the time evolution of the leading and trailing edge displacements is shown in Fig. 3. The amplitude of vertical oscillations of the trailing edge is more than twice $y_{F}$. Also note the phase lag of about $0.11 t_{F}$. Horizontal oscillations of the trailing edge are non-negligible: their amplitude is about $0.5 y_{F}$.

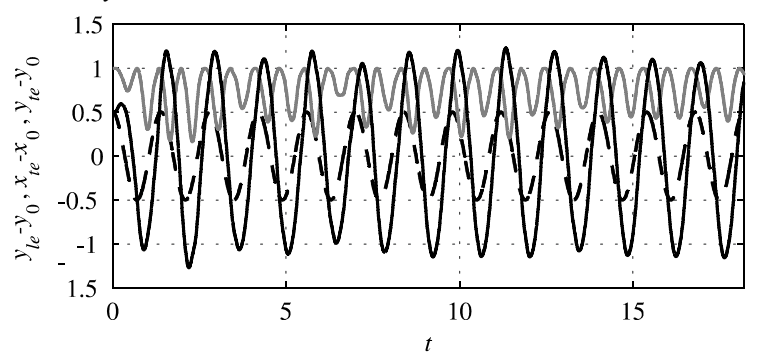

Fig. 3. Time evolution of the vertical displacement of the leading edge $y_{l e}-y_{0}$ (black dashed line), vertical displacement of the trailing edge $y_{t e}-y_{0}$ (black solid line) and horizontal displacement of the trailing edge $x_{t e}-x_{0}$ (grey line).

(a)

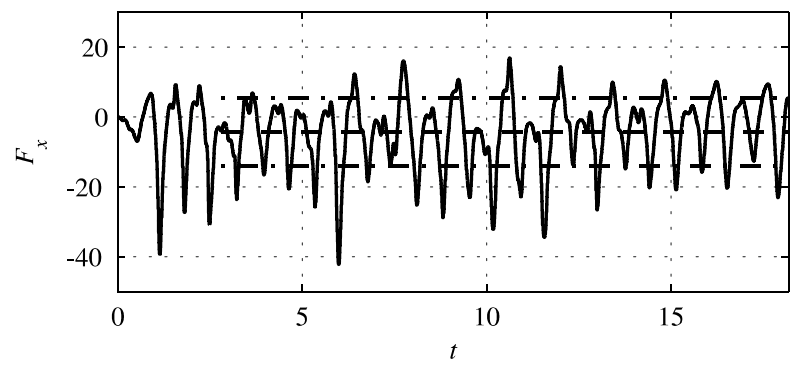

(b)

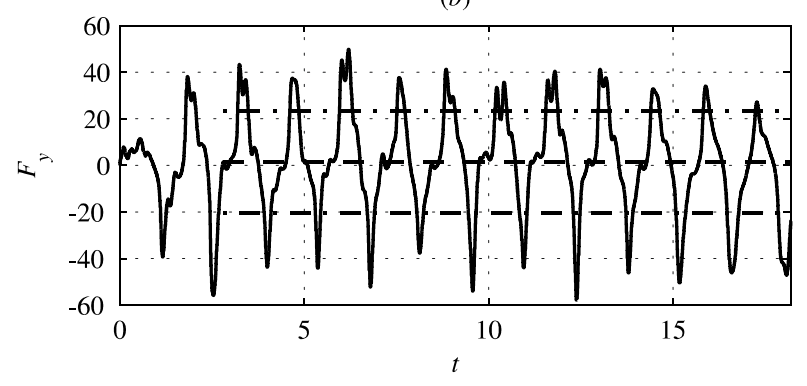

Fig. 4. Time evolution of the horizontal (a) and vertical (b) component of the fluid-dynamic force. Dashed lines show the mean force over the last 11 strokes, and dash-dotted lines indicate intervals of plus/minus one standard deviation. The time span of one stroke is $t_{F}=1.4$ (frequency $f \approx 0.714$ ).

Fig. 4 presents the time evolution of the fluiddynamic force acting on the wing for the case $t_{F}=1.4$. The $x$-component of the force (thrust) oscillates but does not reach a periodic state during the simulation. Peaks occur with twice the stroke frequency. However, after $t=8$, every second peak is stronger, indicating a systematic asymmetry between the upstrokes and downstrokes. The mean value is negative, i.e., the wing generates a non-zero thrust. The $y$-component (lift) oscillates with the stroke frequency. Its mean value 
over the last eleven strokes is approximately zero. However, there is no top-bottom symmetry in the time evolution of the lift, and over each period individually it may have a non-zero average (like over the last period, for example). For a freely flying insect this may result in a slow fluctuation of the body in the vertical direction.

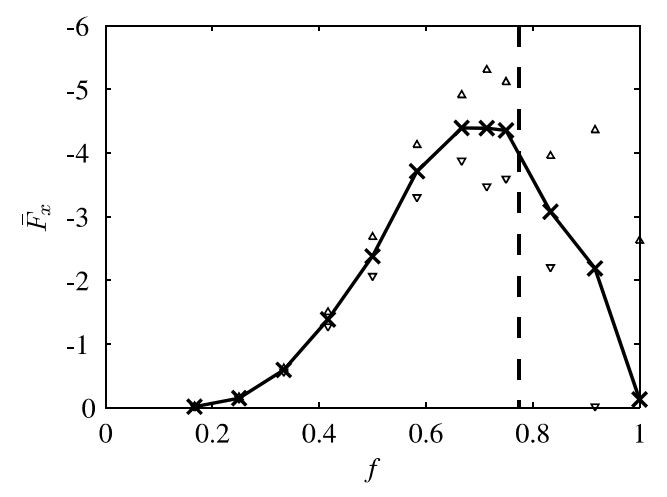

Fig. 5. Mean thrust force $\bar{F}_{x}$ versus stroke frequency $f$.

Triangular markers show $95 \%$ confidence intervals for every simulation point. The dashed line shows the first eigenfrequency $f_{0}=0.77(8)$.

Fig. 5 displays the mean thrust as a function of the stroke frequency $f=1 / t_{F}$. It was estimated by averaging over the last 11 strokes whereas the simulations spanned 13 strokes. When the frequency is small, the thrust increases from zero non-linearly until it reaches its maximum at $\approx \approx 0.714\left(t_{F} \approx 1.4\right)$. Then it rapidly drops at larger frequencies. We notice that in no case the thrust force was exactly periodic in time, therefore the estimate of its mean value could vary appreciably depending on the interval used for averaging. For that reason Fig. 5 also shows, in addition to the mean thrust, the theoretical $95 \%$ confidence intervals for the mean thrust,

$\left[\bar{F}_{x}-\frac{C S}{\sqrt{J}} ; \bar{F}_{x}+\frac{C S}{\sqrt{J}}\right]$, where $S^{2}=\frac{1}{J-1} \sum_{j=1}^{J}\left(F_{x j}-\bar{F}_{x}\right)^{2},(7)$

$C=1.96, \bar{F}_{x}$ is the average force over the last $J=11$ strokes in the simulation and $F_{x j}$ is an average over the $j$-th of those strokes. As frequency increases, the flow becomes more erratic and the mean thrust estimates less accurate. Nevertheless, Fig. 5 provides a strong evidence that the maximum thrust develops between $f=0.65$ and 0.75 . For comparison, the first eigenfrequency of the wing

$$
f_{0}=\frac{3.516}{2 \pi} \sqrt{\frac{\eta}{\mu}}=0.77
$$

is shown in the same figure with a vertical dashed line. This agrees with the experimental results reported in [6] to the extent that the optimal stroke frequency is lower than the resonance frequency, but the difference between them is not as large in our numerical simulations as in the experiment [6]. We remind that, in the experiment, the Reynolds number was larger and the flow was three-dimensional.
Fig. 6 shows the maximum deflection angle at the trailing edge, $\theta_{\text {te } \max }=\max \left|\theta_{\text {te }}\right|$, over the last ten strokes. At $f=0.714$, when the force is near to its maximum, the angle equals $\theta_{\text {te } \max }=106.6^{\circ}$. At larger frequencies, the wing bends so much that the trailing edge points even more forwards, so that the fluid momentum is also directed forwards. Therefore thrust decreases. Note that $\theta_{\text {te } \max }(f)$ is almost a straight line in the frequency range considered in our simulations.

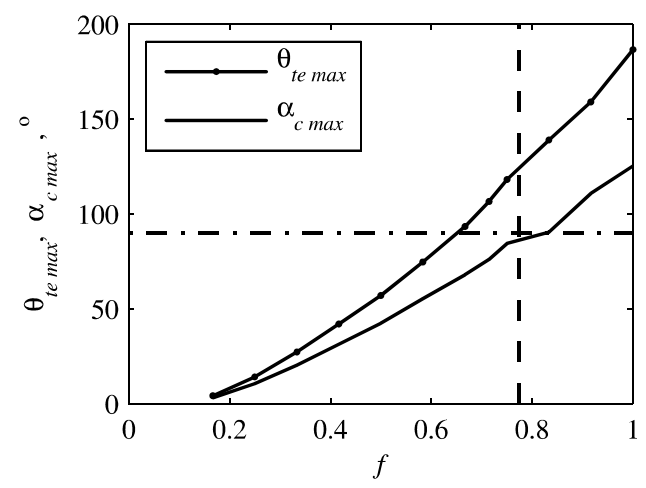

Fig. 6. Maximum deflection angle at the trailing edge $\theta_{t e}$ and maximum value of geometric angle of attack $\alpha_{c}$ versus the stroke frequency $f$. The vertical dashed line shows the first eigenfrequency $f_{0}=0.77$ (8). The horizontal dash-dotted line indicates $90^{\circ}$.

The geometric angle of attack of a flexible foil can be defined as the angle between the instantaneous chord line and the direction of flight. Since in our case the far-field velocity is zero, we use the horizontal direction instead, that is the direction of the propulsive force (see Fig. 1c): $\alpha_{c}=-\arctan \left(\left(y_{t e}-y_{l e}\right) /\left(x_{t e}-x_{l e}\right)\right)$. Maximum absolute values of this angle, $\alpha_{c \max }=\max \left|\alpha_{c}\right|$, versus frequency $f$ are displayed in Fig. 6 . Note that the ratio $\alpha_{c} / \theta_{\text {te max }}$ is bounded between 0.65 and 0.75 .

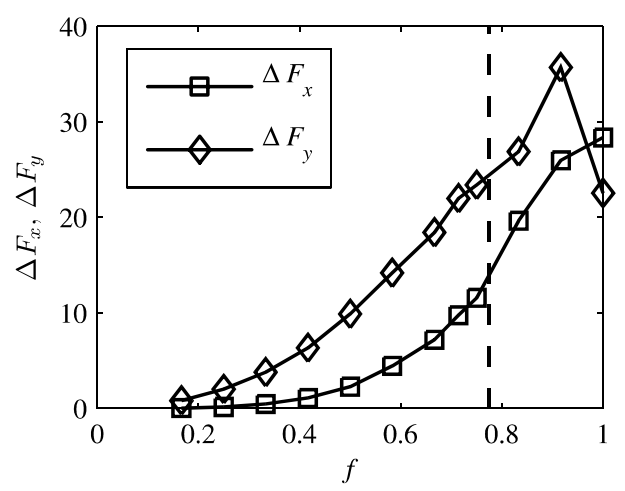

Fig. 7. Standard deviation of the thrust, $\Delta F_{x}$, and of the lift, $\Delta F_{y}$, versus the stroke frequency $f$. The dashed line shows the first eigenfrequency $f_{0}=0.77$ (8).

In all simulations, the average value of the thrust force was relatively small compared to its fluctuating part (see, e.g., Fig. 4). It is convenient to use the standard deviation

$$
\Delta F_{x}=\sqrt{\frac{1}{J t_{F}} \int_{t_{\max }-J t_{F}}^{t_{\max }}\left(F_{x}(t)-\bar{F}_{x}\right)^{2} \mathrm{~d} t}
$$


to evaluate the average magnitude of these fluctuations. The standard deviation of the lift, $\Delta F_{y}$, is defined analogously. Both quantities are shown in Fig. 7 as functions of the stroke frequency. They increase with $f$. A faster increase of $\Delta F_{x}$ at higher frequencies may be related to the increasing deformation of the beam. When the frequency is very high, $f \approx 1$, the time evolution of the force becomes erratic and one may need a longer time interval to determine $\Delta F_{x}$ and $\Delta F_{y}$ reliably. For $t_{F} \approx 1.4$, the intervals of $\pm \Delta F_{x}$ and $\pm \Delta F_{y}$ are indicated with dash-dots in Fig. $4(a)$ and $(b)$, respectively.

The propulsive efficiency $\eta_{0}$ can be defined as a ratio of the propulsive force coefficient to the power coefficient [7]. The power consists of contributions due to the fluid-dynamic force and the inertial force. In our numerical simulations, the integral inertial force of the wing was not calculated, but it was estimated during post-processing as $F_{\text {inert }}=-\mu \mathrm{d}^{2} y_{c g} / \mathrm{d} t^{2} \quad$, where $y_{c g}=y_{l e}+0.375\left(y_{t e}-y_{l e}\right)$. This allowed an approximate estimate of the maximum propulsive efficiency, $\eta_{0 \max }=0.095$ that is achieved at $f \approx 0.7 f_{0}$.

\section{B. Two wing sections}

A series of numerical simulations have been carried out to explore the effect of aerodynamic interactions between two foils. This configuration corresponds to the wings of dragonflies. The motion of the forewing is the same as described in the previous section. The hindwing is placed behind the forewing as indicated in Fig. 2. Its geometrical and material properties are the same. The position of its leading edge varies in time as $x_{l e 2}=x_{0}+d$ and $y_{l e 2}=y_{0}+y_{F} \cos \left(2 \pi t / t_{F}+\delta\right)$. We carried out numerical simulations for the phase shift equal to $\delta=0$ (in phase), $0.25 \pi, 0.5 \pi, 0.75 \pi, \pi$ (antiphase), $1.25 \pi$, $1.5 \pi$ and $1.75 \pi$. The spacing between the leading edges is $d=1.1$. The amplitude is the same for both wings, $y_{F}=0.5$, and the oscillation period is $t_{F}=1.4$. The results of these computations are summarized in Fig. 8. It shows the mean thrust generated by the two wings, $\bar{F}_{x \Sigma}$, the mean thrust of the forewing, $\bar{F}_{x 1}$, and of the hindwing, $\bar{F}_{x 2}$. All of them significantly depend on $\delta$. When the two foils are in phase, $\delta=0$, the total thrust $\bar{F}_{x \Sigma}$ is approximately twice as large as the thrust of a single foil reported above. However, the forewing generates a significantly larger force than the hindwing. This aerodynamic interference is similar to the 'anomalous hydrodynamic drafting' observed by Ristroph and Zhang in experiments with a pair of tandem flags [10]. The total thrust $\bar{F}_{x \Sigma}$ drops to -1.96 when the hindwing is leading $(\delta=0 \ldots \pi)$ and then restores after $\delta=\pi$. When the forewing is leading $(\delta=\pi \ldots 2 \pi), \bar{F}_{x \Sigma}$ reaches its peak value of -10.6 at $\delta=1.25 \pi$. In most cases, the forewing generates larger thrust than the hindwing. However, this is not the case at $\delta=1.25 \pi$, when $\bar{F}_{x 2}$ has a sharp peak which results in the maximum $\bar{F}_{x \Sigma}$.

The observed maximum-thrust phase shift $\delta=1.25 \pi$ is consistent with results of earlier studies of dragonfly flight [8] (since the reference considers a slow-climbing flight, $\bar{F}_{x \Sigma}$ should be compared to the component of the aerodynamic force perpendicular to the stroke plane). However, we find a much larger variation of $\bar{F}_{x \Sigma}$ with $\delta$, which can be explained by differences in the modelling of wing-wake interaction. The present approach accounts for the dynamics of spanwise vortices and their interaction with the foils. On the other hand, the two-dimensional approximation may tend to overestimate the role of these structures, while neglecting the important effect of wing-tip vortices.

A possible reason for the large propulsive force at $\delta=1.25 \pi$ could be a favourable interaction between the hindwing and the vortices shed from the forewing. Fig. 9 displays the vorticity field at four different time instants. In Fig. 9(a), two counter-clockwise vortices are present near the trailing edge of the forewing. Vortex $A$ is the trailing-edge vortex generated during downstroke. Vortex $B$ is the leading-edge vortex generated during the preceding upstroke. One can follow the time evolution of these vortices in Fig. 9(b)$9(d)$. First, vortex $B$ merges with the nearby vorticity sheet and then approaches the leading edge of the hindwing. Later, vortex $A$ impinges the leading edges. Both events increase the pressure deficit at the leading edge, thus resulting in an extra propulsive force. Vortex $B$ may also have some negative effect when it goes further downstream (Fig. 9d), but its strength decreases by that time. This wake capture process occurs at every half-stroke, therefore it is likely to make a significant contribution to the mean thrust. However, a quantitative estimate of the importance of this mechanism does not follow directly from our simulations.

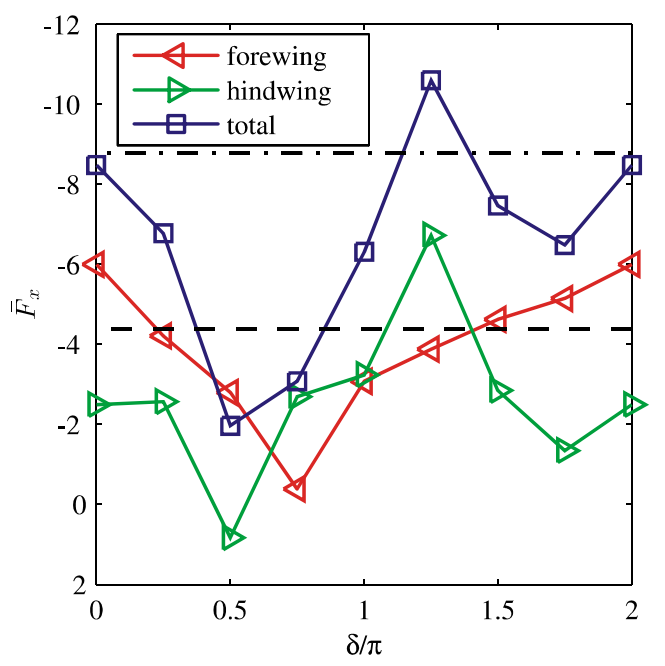

Fig. 8. Mean thrust force $\bar{F}_{x}$ of two foils versus phase shift $\delta$. Dashed line indicates the thrust of a single foil. Dashdotted line shows the same value doubled. 

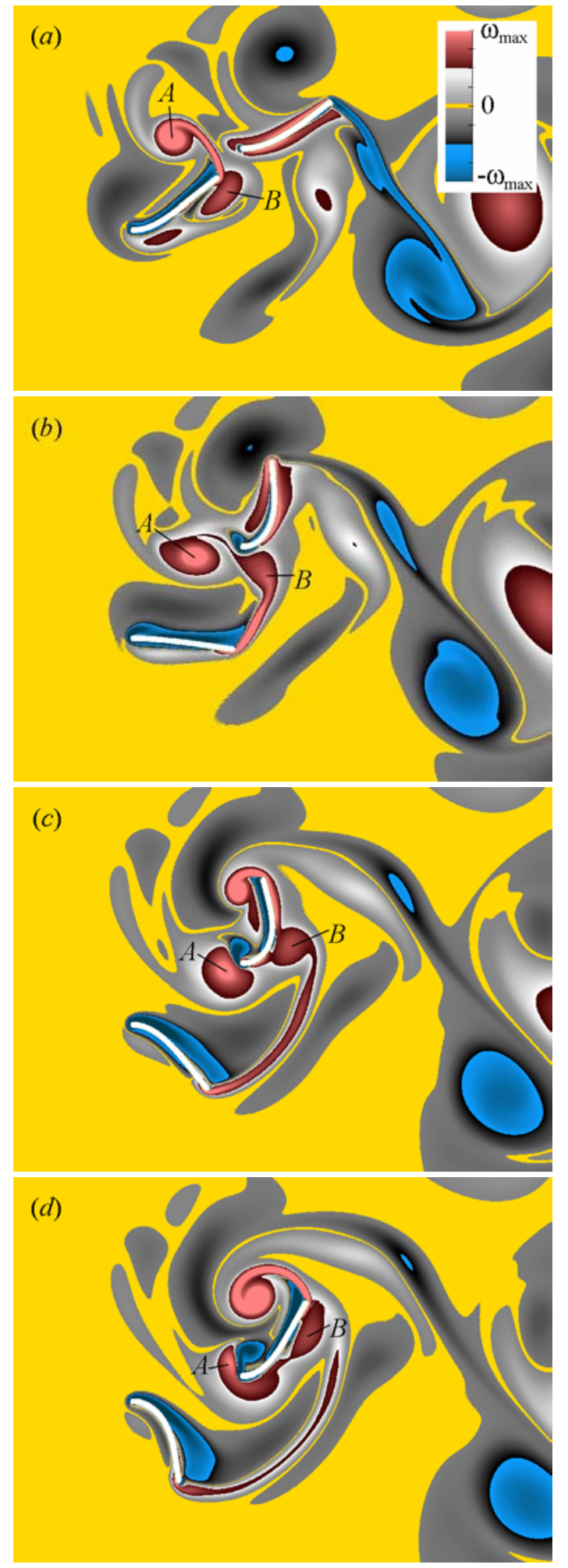

Fig. 9. Vorticity field of two foils flapping with phase difference $\delta=1.25 \pi$. Time instants are $t=14.5(a), 14.65(b)$, $14.8(c)$ and $14.9(d)$

In addition to the mean forces, Table 1 shows the standard deviations, trailing edge deflection angles, and geometric angle of attack in some cases: $\delta=0$ (in phase), $\delta=1.25 \pi$ (maximum thrust) and $\delta=\pi$ (antiphase). The maximum deflection angle at the trailing edge is such that $\theta_{\text {te } \max 1}>\theta_{\text {te } \max \text { single }}>\theta_{\text {te } \max 2}$ when $\delta=0$ and $\theta_{\text {te } \max 1}<$ $\theta_{\text {te } \max \text { single }}<\theta_{\text {te } \max 2}$ when $\delta=1.25 \pi$ or $\delta=\pi$. Subscripts '1' and ' 2 ' correspond to the forewing and the hindwing, respectively, and $\theta_{\text {te } \max \text { single }}=106.6^{\circ}$ is the angle at the trailing edge of a single foil flapping with the same frequency (see Fig. 6).

The standard deviation of the total lift, $\Delta F_{y \Sigma}$, is the smallest when $\delta=\pi$, because the frequency of oscillation of $F_{y 1}$ and $F_{y 2}$ is equal to $f$. This consideration may be important for minimizing the body vibration in free flight.

Also note that, in a free flight, it is important to ensure that the pitching moment acting on the body is zero. This is the reason why a dragonfly chooses $\delta \approx 80^{\circ}$ despite some loss in the aerodynamic performance [8]. Meanwhile, for a micro air vehicle, it may be possible to design wings such that the pitching moment vanishes at the same $\delta$ as the propulsive force is maximized.

The rightmost column in Table 1 shows some results of a numerical simulation with a different value of spacing between the leading edges, $d=1.6$, in the antiphase case only. The unfavorable aerodynamic interaction becomes weaker compared to the previous case, $d=1.1$, and the forces of both wings approach the values corresponding to a single foil reported in Section IV.A.

\begin{tabular}{|l|l|l|l|l|}
\hline$d$ & 1.1 & 1.1 & 1.1 & 1.6 \\
\hline$\delta$ & 0 & $1.25 \pi$ & $\pi$ & $\pi$ \\
\hline $\bar{F}_{x 1}$ & $-6.00 \pm 1.04$ & $-3.88 \pm 0.84$ & $-3.07 \pm 0.77$ & $-3.66 \pm 1.27$ \\
\hline $\bar{F}_{x 2}$ & $-2.49 \pm 1.07$ & $-6.73 \pm 1.08$ & $-3.24 \pm 1.66$ & $-3.63 \pm 1.06$ \\
\hline $\bar{F}_{x \Sigma}$ & $-8.49 \pm 1.70$ & $-10.6 \pm 1.64$ & $-6.31 \pm 2.11$ & $-7.29 \pm 2.05$ \\
\hline$\Delta F_{x 1}$ & 10.26 & 8.19 & 8.62 & 8.82 \\
\hline$\Delta F_{x 2}$ & 11.52 & 12.18 & 10.81 & 10.85 \\
\hline$\Delta F_{x \Sigma}$ & 20.16 & 10.84 & 14.62 & 16.05 \\
\hline$\Delta F_{y 1}$ & 25.75 & 21.51 & 21.91 & 20.37 \\
\hline$\Delta F_{y 2}$ & 26.54 & 17.65 & 19.60 & 17.98 \\
\hline$\Delta F_{y \Sigma}$ & 51.28 & 25.50 & 19.41 & 14.60 \\
\hline$\theta_{t e \max 1}$ & $112.6^{\circ}$ & $99.1^{\circ}$ & $100.5^{\circ}$ & $107.8^{\circ}$ \\
\hline$\theta_{t e \max 2}$ & $89.7^{\circ}$ & $116.7^{\circ}$ & $122.6^{\circ}$ & $114.1^{\circ}$ \\
\hline$\alpha_{c \max 1}$ & $79.2^{\circ}$ & $70^{\circ}$ & $71.4^{\circ}$ & $77.8^{\circ}$ \\
\hline$\alpha_{c \max 2}$ & $65.2^{\circ}$ & $84.2^{\circ}$ & $89.4^{\circ}$ & $80.2^{\circ}$ \\
\hline
\end{tabular}

Table 1. Horizontal spacing between the leading edges $d$, phase shift $\delta$, mean thrust $\bar{F}_{x}$, standard deviation of thrust $\Delta F_{x}$, and of lift $\Delta F_{y}$,

maximum magnitude of the deflection angle $\theta_{\text {te } \max }$ at the trailing edge, maximum magnitude of the geometric angle of attack $\alpha_{c \max }$ of the foils in the two-wing configuration. Subscripts 1, 2 and $\Sigma$ stand for the forewing, the hindwing and the total, respectively.

\section{CONCLUSIONS}

A new method for numerical simulation of viscous incompressible fluid flows past flexible flapping wings has been developed. It allows modelling of single or multiple wing sections. 
Numerical simulations of a single flapping foil indicate that the maximum thrust is achieved at a stroke frequency slightly lower than the lowest eigenfrequency of the wing, which is in agreement with experimental findings [6]. The maximum deflection angle at the trailing edge equals $106.6^{\circ}$ at the maximum thrust regime, and its further increase with the frequency results in a decrease of the mean thrust. The oscillatory part of the force only increases in amplitude.

Numerical simulations of two foils show that, at the stroke frequency $f=0.714$ (which was found to maximize the thrust of a single foil), two foils flapping with a phase shift $\delta=1.25 \pi$ generate the maximum propulsive force. We also observe an 'anomalous hydrodynamic drafting' when the foils flap in phase, $\delta=0$.

\section{ACKNOWLEDGEMENTS}

We thank R. Godoy-Diana for enlightening discussions. TE and KS acknowledge financial support from the DFH-UFA.

\section{References}

[1] M.S. Triantafyllou, A.H. Techet and F.S.Hover, "Review of experimental work in biomimetic foils," IEEE Journal of Oceanic Engineering, vol. 29, pp. 585-594, 2004.

[2] K. Schneider, "Numerical simulation of the transient flow behaviour in chemical reactors using a penalisation method," Computers and Fluids, vol. 34, pp. 1223-1238, 2005.

[3] D. Kolomenskiy and K. Schneider, "A Fourier spectral method for the Navier-Stokes equations with volume penalization for moving solid obstacles," Journal of Computational Physics, vol. 228, pp. 5687-5709, 2009.

[4] T. Engels, D. Kolomenskiy, K. Schneider and J. Sesterhenn, "Numerical simulation of the fluttering instability using a pseudospectral method with volume penalization," Computers and Structures, in press.

[5] P. Angot, C.-H. Bruneau and P. Fabrie, "A penalization method to take into account obstacles in incompressible viscous flows," Numerische Mathematik, vol. 81, pp. 497520, 1999.

[6] S. Ramananarivo, R. Godoy-Diana and B. Thiria, "Rather than resonance, flapping wing flyers may play on aerodynamics to improve performance," Proceedings of the National Academy Sciences of the United States of America, vol. 108, pp. 5964-5969, 2011.

[7] C.-K. Kang, H. Aono, C.E.S. Cesnik and W. Shyy, "Effects of flexibility on the aerodynamic performance of flapping wings," Journal of Fluid Mechanics, vol. 689, pp. 32-74, 2011.

[8] A. Azuma, S. Azuma, I. Watanabe and T. Furuta, "Flight mechanics of a dragonfly," Journal of Experimental Biology, vol. 116, pp. 79-107, 1985.

[9] T. Nakata and H. Liu, "A fluid-structure interaction model of insect flight with flexible wings," Journal of Computational Physics, vol. 231, pp. 1822-1847, 2012.

[10] L. Ristroph and J. Zhang, "Anomalous hydrodynamic drafting of interacting flapping flags," Physical Review Letters, vol. 101, pp. 194502 1-4, 2008. 